\title{
Everolimus improves neuropsychiatric symptoms in a patient with tuberous sclerosis carrying a novel TSC2 mutation
}

\author{
Su-Kyeong Hwang ${ }^{1 \dagger}$, Jae-Hyung Lee ${ }^{2 \dagger}$, Jung-eun Yang ${ }^{3 \dagger}$, Chae-Seok Lim ${ }^{3 \dagger}$, Jin-A Lee ${ }^{4}$, Yong-Seok Lee ${ }^{5^{*}}$,
} Kyungmin Lee ${ }^{6^{*}}$ and Bong-Kiun Kaang ${ }^{3^{*}}$

\begin{abstract}
Tuberous sclerosis complex (TSC) is a neurocutaneous disorder characterized by multiple symptoms including neuropsychological deficits such as seizures, intellectual disability, and autism. TSC is inherited in an autosomal dominant pattern and is caused by mutations in either the TSC1 or TSC2 genes, which enhance activation of the mammalian target of rapamycin (mTOR) signaling pathway. Recent studies have suggested that mTOR inhibitors such as rapamycin can reverse TSC-associated deficits in rodent models of TSC. In addition, clinical trials are ongoing to test the efficacy of mTOR inhibitors toward the psychiatric symptoms associated with TSC. Here, we report a case study of a Korean patient with TSC, who exhibited multiple symptoms including frequent seizures, intellectual disability, language delays, and social problems. We performed whole exome sequencing and identified a novel small deletion mutation in TSC2. Expressing the novel deletion mutant in HEK293T cells significantly increased mTOR pathway activation. Furthermore, everolimus treatment showed not only reduction in SEGA size, but dramatically improved behavioral deficits including autism related behaviors in the patient. In summary, we identified a novel small deletion mutation in TSC2 associated with severe TSC in a Korean family that enhances the activation of mTOR signaling in vitro. Everolimus treatment improved behavioral deficits in the patient.
\end{abstract}

Keywords: Tuberous sclerosis, Autism, Everolimus, Mutation, High throughput nucleotide sequencing

\section{Introduction}

Tuberous sclerosis complex (TSC) is a genetic disorder characterized by the presence of benign hamartomas in any organ system, with highly variable, unpredictable, and potentially devastating neurological outcomes $[1,2]$. TSC is the second most common identified neurocutaneous disorder with an estimated incidence of 1:6000, affecting more than 1 million individuals worldwide $[3,4]$. The majority of patients have central nervous system involvement that manifests as structural

\footnotetext{
*Correspondence: yongseok@cau.ac.kr; iriskim@knu.ac.kr; kaang@snu.ac.kr ${ }^{\dagger}$ Equal contributors

${ }^{5}$ Department of Life Science, Chung-Ang University, Seoul 06974, South Korea

${ }^{6}$ Behavioral Neural Circuitry and Physiology Laboratory, Department of Anatomy, Brain Science \& Engineering Institute, Kyungpook National University Graduate School of Medicine, Daegu 41944, South Korea ${ }^{3}$ Department of Biological Sciences, College of Natural Sciences, Seoul National University, Seoul 08826, South Korea

Full list of author information is available at the end of the article
}

brain abnormalities, epilepsy, and cognitive, behavioral, and psychiatric deficits including autism spectrum disorder (ASD) [5, 6]. All types of seizures are seen, often in combination. Half of individuals have normal intelligence, but almost none are free from neuropsychiatric problems [7]. Importantly, ASD is diagnosed in approximately $40-60 \%$ of patients with TSC, and TSC accounts for $3-4 \%$ of ASD [8-10].

TSC is caused by mutations in either of two tumor suppressor genes, TSC1 or TSC2, encoding hamartin and tuberin, respectively [11]. TSC is inherited in an autosomal dominant pattern and haploinsufficiency of TSC causes neurological phenotypes including learning disability and social deficits $[10,11]$. De novo mutations account for approximately $80 \%$ of TSC cases. TSC $2 \mathrm{mu}-$ tations are four times as common as TSC1 mutations among de novo cases, whereas the prevalence of TSC1 and TSC2 mutations is approximately equal among familial TSC cases [12]. TSC1 and TSC2 mutations lead to 
essentially identical phenotypic manifestations, although there have been some suggestions that the TSC2 phenotype is typically more severe $[13,14]$. The TSC1 and TSC2 proteins act as a heterodimer to suppress mammalian target of rapamycin (mTOR), a serine/threonine protein kinase that regulates cell growth and division [5, 15]. Loss of either TSC1 or TSC2, followed by a "second hit" of the remaining functional allele thereby preventing formation of the heterodimeric complex, causes loss of regulatory control over mTOR and leads to overactive cell growth and proliferation $[1,16]$. Thus, at the cellular level, loss of TSC1 or TSC2 results in upregulation of the mTOR pathway $[10,17]$. The molecular understanding of the TSC pathophysiology has opened up possibilities for molecular targeted treatments of the neuropsychiatric phenotype in TSC using mTOR inhibitors such as rapamycin [16]. Notably, rapamycin treatments have been shown to successfully reverse the deficits in behavior and synaptic plasticity in rodent models of TSC [10, 18-20].

Recently, the mTOR inhibitors everolimus and sirolimus have been shown to exhibit efficacy for the treatment of several manifestations of TSC such as subependymal giant cell astrocytomas (SEGA), seizures, renal angiomyolipomas, lymphangioleiomyomatosis, and facial angiofibroma lesions in patient with TSC [21-24]. Moreover, human and animal studies suggest that mTOR inhibitors improve deficits of sociability, learning and neurodevelopment in TSC mouse models and patients with TSC $[18,25,26]$. On the basis of these findings, although some clinical trials have been completed or initiated to test whether everolimus treatment might improve neurocognition, features of autism, and the neuropsychological deficits in children with TSC (clinicaltrials.gov study ID: NCT01289912, NCT01730209), in the present study we present a case of a family with a novel TSC2 mutation in which the behavioral phenotypes of a 3-year-old boy with TSC accompanied by severe autism could be dramatically improved by everolimus treatment.

\section{Results}

\section{Subject characteristics}

The proband presented intractable epilepsy and severe developmental delay. He was born at 38 weeks gestation by spontaneous vaginal delivery with a birth weight of $2.4 \mathrm{~kg}$ after an uncomplicated pregnancy. At the age of 13 months, he experienced his first episode of febrile status epilepticus with a duration of $40 \mathrm{~min}$; at 17 months, a second episode of febrile status epilepticus persisted for more than an hour. Subsequently, he had frequent seizures with or without fever, and was often admitted with status epilepticus. Physical examination identified scattered hypopigmented lesions on the trunk
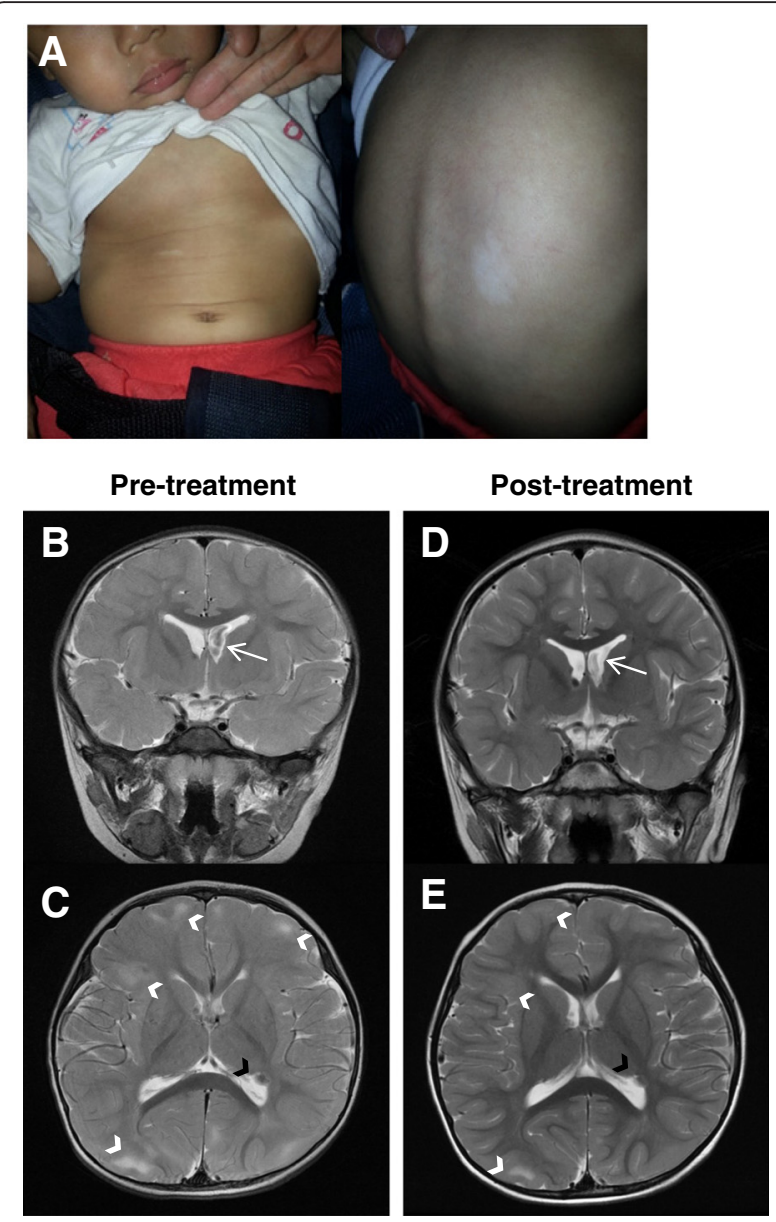

Fig. 1 Cutaneous features and brain MRI findings of the patient. a Photographs showing several hypopigmented macules on the chest and abdomen (left) and on the back (right) of the patient. b-c. Pre-treatment brain MRI revealing a SEGA (white arrow) located at the foramen of Monro within on coronal T2-weighted imaging (b), multiple cortical tubers (white arrowheads) and a subependymal nodule (black arrowhead) within on axial T2-weighted imaging (c). d-e Post-treatment brain MRI demonstrating size reduction of the SEGA (white arrow) (d), reduced cortical tubers (white arrowheads) and the same size of subependymal nodule (black arrowhead) (e) compared to pre-treatment MRI

(Fig. 1a). Brain magnetic resonance imaging (MRI) revealed a SEGA (Fig. 1b), cortical tubers and subependymal nodules consistent with TSC (Fig. 1c). The seizures persisted despite the use of vigabatrin and levetiracetam at the maximum tolerated doses.

A baseline psychomotor developmental evaluation was performed when the patient was 45 months of age. The Denver Developmental Screening Test (DDST-II) revealed delayed development in areas of fine motor-adaptive, personal-social, and language skills by 25 months on average. He was not able to get a score in any subtests in the Wechsler Preschool and Primary Scale of Intelligence 
(WPPSI-III). His social age was 22 months and social quotient was 49.8 by the Social Maturity Scale (SMS). The Childhood Autism Rating Scale (CARS) and Autism Diagnostic Observation Schedule-2 (ADOS-2) showed scores of 35.5 and 20, respectively, which were above the cutoff scores for a diagnosis of autism. His Autism Diagnostic Interview-Revised (ADI-R) scores were also above the autism diagnostic cutoffs in each criterion; 24 in qualitative abnormalities in reciprocal social interaction, 18 for verbal and 12 for non-verbal in qualitative abnormalities in communication, and 5 in repetitive/stereotyped patterns of behavior. The speech and language evaluation revealed that his receptive and expressive language was delayed by 27 months. Other evaluations including ophthalmologic examination, dental examination, ultrasonography and electrocardiography (ECG) of the heart, high resolution

Table 1 Evaluations for TSC diagnosis

\begin{tabular}{|c|c|c|}
\hline $\begin{array}{l}\text { Organ } \\
\text { system }\end{array}$ & Diagnostic evaluations & Findings \\
\hline \multirow[t]{8}{*}{ Brain } & Brain MRI & $\begin{array}{l}\text { Multiple cortical tubers, } \\
\text { subependymal nodules and a } \\
\text { SEGA }\end{array}$ \\
\hline & EEG & $\begin{array}{l}\text { Runs of spikes in right fronto- } \\
\text { polar region }\end{array}$ \\
\hline & DDST-II & $\begin{array}{l}\text { Delayed development in areas } \\
\text { of fine motor-adaptive, } \\
\text { personal-social, and language } \\
\text { skills by } 25 \text { months on average }\end{array}$ \\
\hline & WPPSI-III & $\begin{array}{l}\text { Could not get a score in any } \\
\text { subtest }\end{array}$ \\
\hline & CARS & 35.5 \\
\hline & \multirow[t]{2}{*}{ SMS } & Social age: 22 months \\
\hline & & Social quotient: 49.8 \\
\hline & $\begin{array}{l}\text { Speech and language } \\
\text { evaluation }\end{array}$ & $\begin{array}{l}\text { Delayed by } 27 \text { months in } \\
\text { receptive and expressive } \\
\text { language }\end{array}$ \\
\hline Eyes & $\begin{array}{l}\text { Complete ophthalmologic } \\
\text { evaluation including dilated } \\
\text { fundoscopy }\end{array}$ & Unremarkable \\
\hline Teeth & Detailed dental exam & Unremarkable \\
\hline Skin & Detailed skin exam & $\begin{array}{l}\text { Scattered hypopigmented } \\
\text { lesions }\end{array}$ \\
\hline \multirow[t]{2}{*}{ Heart } & Ultrasonography & $\begin{array}{l}\text { Small muscular ventricular } \\
\text { septal defect (VSD) in the } \\
\text { middle portion of the } \\
\text { interventricular septum (IVS), } \\
\text { No rhabdomyoma }\end{array}$ \\
\hline & ECG & Normal \\
\hline Lung & HRCT of chest & $\begin{array}{l}\text { No lymphangioleiomyomatosis } \\
\text { (LAM) }\end{array}$ \\
\hline \multirow[t]{3}{*}{ Kidney } & Blood pressure & Normal \\
\hline & Abdominal MRI & $\begin{array}{l}\text { No angiomyolipoma or renal } \\
\text { cyst }\end{array}$ \\
\hline & GFR test & Normal \\
\hline
\end{tabular}

computed tomography (HRCT) of the chest, blood pressure, abdominal MRI, and glomerular filtration rate (GFR) testing demonstrated no abnormalities. Evaluations for the diagnosis of TSC and the respective findings are listed in Table 1.

\section{Identification of a small deletion variant located in TSC2 by whole exome sequencing}

In the family, three individuals including the grandmother (I-1), father (II-1), and the proband (third son, III-2) met diagnostic criteria for TSC. Definite diagnosis is made by 2 major features or 1 major feature with 2 or more minor features; the grandmother (I-1) and father (II-1) had 3 major features: multiple hypomelanotic macules, angiofibromas, and ungual fibromas. The other family members (including II-2, III-1 and III-3) had no features of TSC (Fig. 2a). In contrast to the proband (III2), the grandmother (I-1) and father (II-1) had normal intelligence and epilepsy or neuropsychiatric symptoms were not identified.

To confirm a TSC gene mutation, we performed whole exome sequencing for the six family members, generating over 50 million reads (average mean target depth: $65 \times$ ). In each family member, about 80,000 variants were identified in RefSeq exonic regions. After filtering common variants (see Methods), over 500 variants causing non-synonymous changes, frameshifts, or which were located near splicing sites were identified in each family member. To identify rare and novel causative candidate variants, we selected the variants that were detected in affected (I-1, II-1, and III-2) but not unaffected family members (II-2, III-1, and III-3). A total of 14 variants were identified, five of which were novel (i.e., not reported in dbSNP142) (Additional file 1: Table S1). Among those five, a variant in GLI2 (NM_004270), c.C2740T (p.R914W) was predicted as deleterious by three different tools (SIFT, PolyPhen-2, and MutationTaster) commonly used to predict the possible impact of amino acid changes. GLI2 is known as a Kruppel-like transcription factor and is associated with cancer progression and metastasis [27]. Notably, one of the novel variants was located in the TSC2 gene, the mutation of which causes TSC [28] and in which over 700 mutations have been reported [29]. We confirmed that only the affected family members (I-1, II-1, and III-2) carried the variant, identified as a small c.700-701 deletion (chr16: 2106697-2016698) by Sanger sequencing (Fig. 2b). The small deletion variant is located in the TSC1 binding domain and the flanking region of the variant is highly conserved across different species (Fig. 2c). We predicted that the deletion could generate a truncated form of the TSC2 protein because of the introduction of a premature termination codon. 


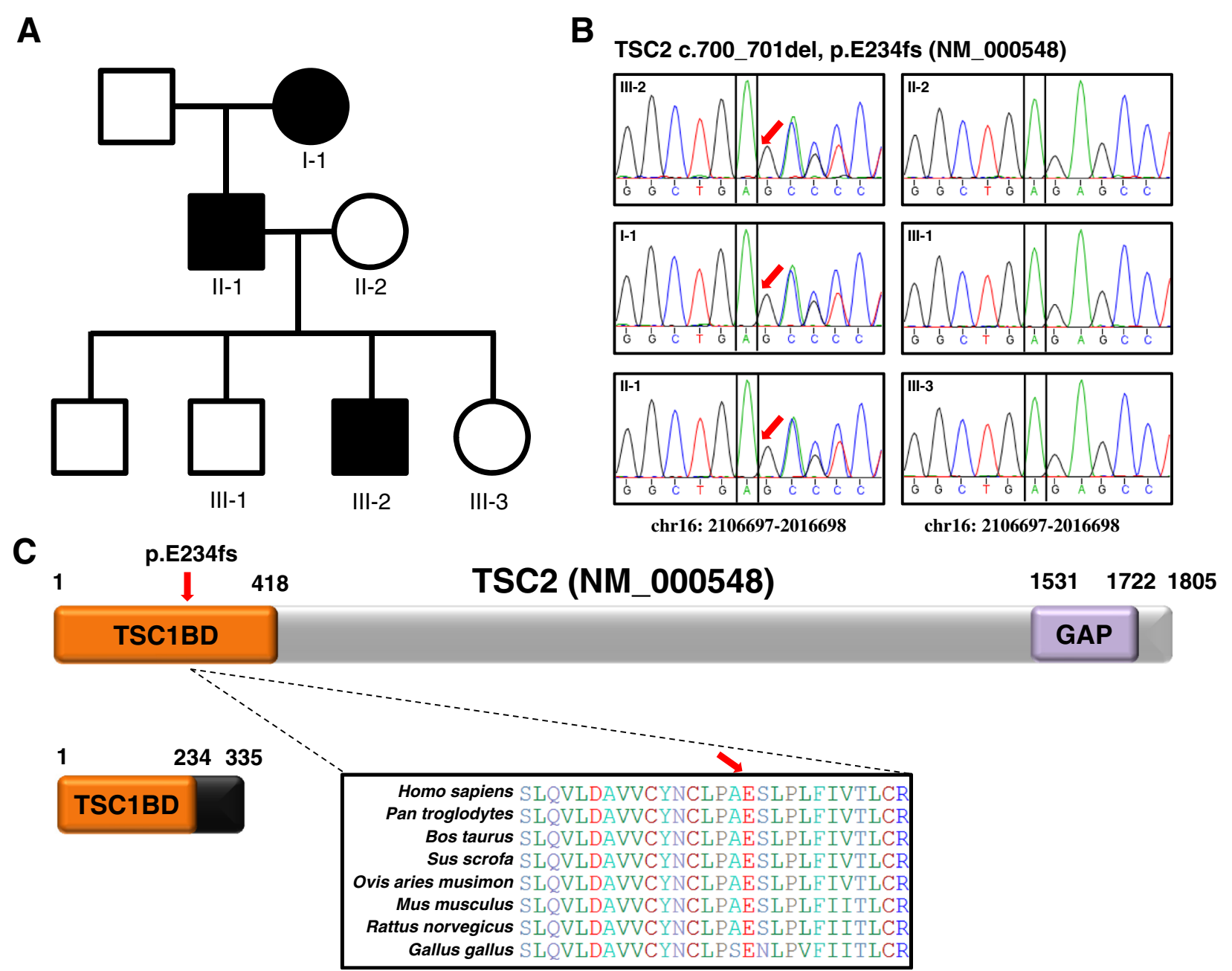

Fig. 2 Identification of a small deletion variant in the TSC2 gene. a Pedigree of the affected family. Closed symbols represent affected family members (I-1, II-1, and III-2). Whole exome sequencing was performed for six family members (I-1, II-1, II-2, III-1, III-2, and III-3). b Confirmation of the identified small deletion variant in the TSC2 gene. The two nucleotide deletion (c.700-701del, chr16: 2106697-2016698) identified in the three affected members was validated by Sanger sequencing of the genomic DNA from six family members. The TSC2 transcript, NM_000548 (RefSeq sequence) with the two nucleotide deletion (c.700-701 del; cDNA position 700 and 701) could be translated into a truncated form of the TSC2 protein because of the frameshift at the amino acid position 234, glutamic acid (p.E234fs). c Schematic diagram of the TSC2 protein with the position of the small deletion identified in this study. The variant is located in the TSC1 binding domain (TSC1BD, orange); it is predicted that the variant generates a premature stop codon in the TSC1BD domain. An amino acid multiple sequence alignment of the region near the small deletion for eight different species shows that the region is evolutionally highly conserved. GAP GTPase activating protein domain

\section{Everolimus treatment rescued abnormal mTOR pathway} activation by the novel TSC2 frameshift mutation

To investigate the effect of the novel frameshift mutation of TSC 2 on mTOR pathway activation, we performed a transfection-based immunoblot assay [30, 31]. As predicted, the novel TSC2 c.700-701 deletion mutant was detected as an approximately $37 \mathrm{kDa}$-sized band, whereas the wild-type TSC2 was detected as an approximately $200 \mathrm{kDa}$-sized band on the blot owing to the frameshift resulting in a premature termination of translation (Fig. 3a). The expression level of TSC2 p.R611Q mutant and c.700-701 deletion mutant was reduced compared to TSC2 wild-type (WT: $1.000 \pm 0.049$, TSC2
p.R611Q mutant: $0.455 \pm 0.065$, TSC2 c.700-701 deletion mutant: $0.040 \pm 0.069$, One-way ANOVA followed by Dunnett's test, all $p<0.0001, n=9$, Fig. 3a and b). Furthermore, the expression level of TSC1 was also reduced compared to that of TSC1 co-transfected with wild-type TSC2 despite the same quantity of TSC1 DNA construct was transfected (pcDNA3.1(+): $0.367 \pm 0.141$, WT: $1.000 \pm 0.041$, TSC2 p.R611Q mutant: $0.161 \pm 0.061$, TSC2 c.700-701 deletion mutant: $0.247 \pm 0.100$, Oneway ANOVA followed by Dunnett's test, all $p<0.0001$, $n=9$, Fig. $3 \mathrm{a}$ and $\mathrm{c}$ ), which is consistent with previous reports [30, 31]. In contrast, the phospho-S6K (T389) level was significantly increased in the novel mutant 


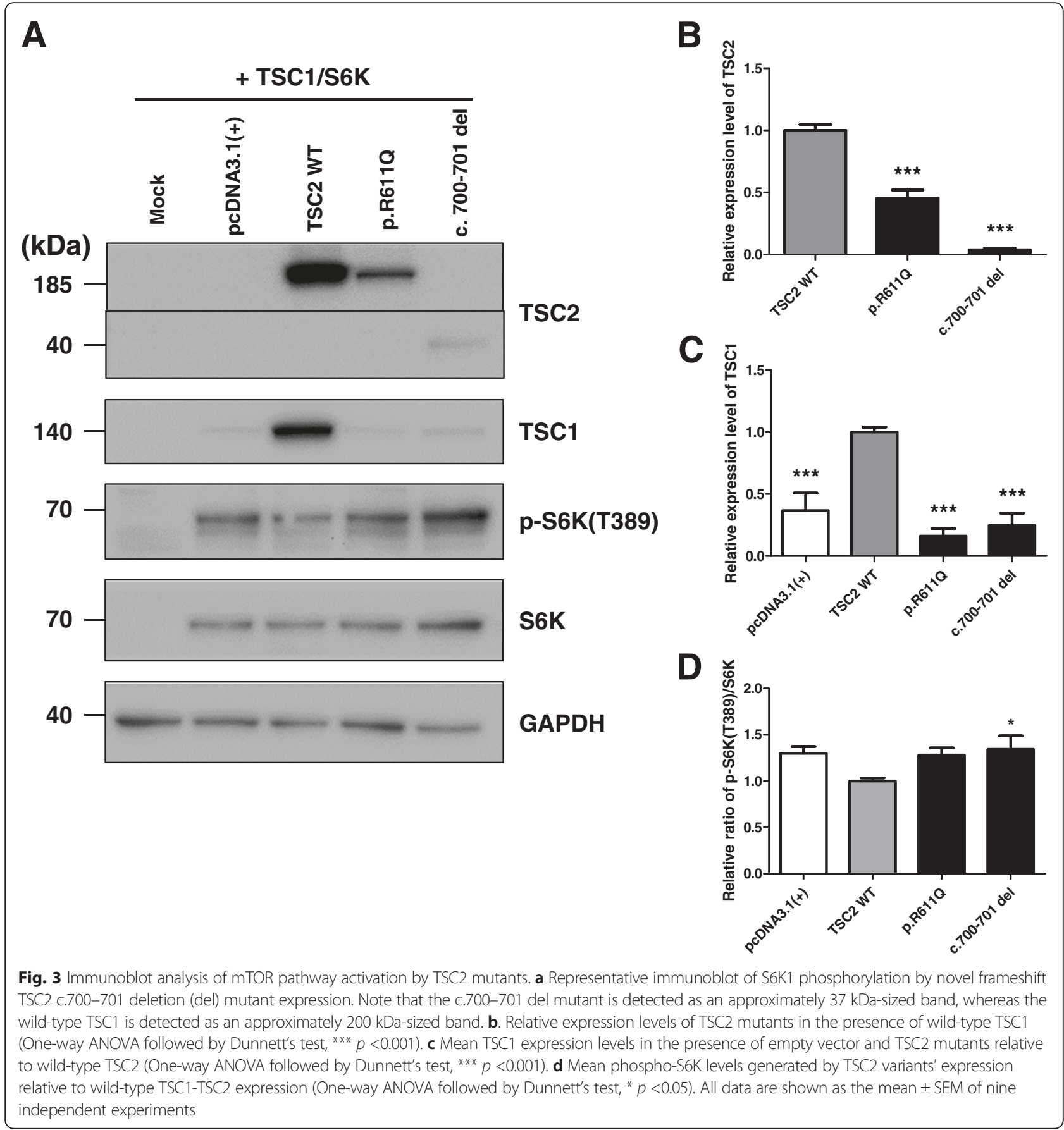

group compared to control wild-type group (WT: 1.000 \pm 0.034 , TSC2 c.700-701 deletion mutant: $1.343 \pm 0.145$, One-way ANOVA followed by Dunnett's test, $p<0.05, n$ $=9$, Fig. $3 \mathrm{a}$ and $\mathrm{d}$ ), suggesting that the expression of the TSC2 c.700-701 deletion mutant results in hyperactivation of the mTOR signaling pathway [30].

Next, to investigate whether an mTOR inhibitor could rescue elevated mTOR pathway activation by the novel TSC2 frameshift mutation, we treated $10 \mathrm{nM}$ everolimus to the TSC2-transfected HEK293T cell cultures and performed immunoblot assays. Everolimus significantly reduced the elevated phospho-S6K (T389) level in p.R611Q mutant group $(4.096 \pm 0.884$ to $0.637 \pm 0.156$, One-way ANOVA followed by Bonferroni's test, $p<0.01, n=3$ ) and novel TSC 2 mutant group (4.285 \pm 0.343 to $0.639 \pm 0.114$, One-way ANOVA followed by Bonferroni's test, $p<0.01 n=3$, Fig. 4a \& b). The phospho-S6K (T389) level in the everolimus-treated wild-type group was not significantly reduced $(1.000 \pm$ 0.241 to $0.693 \pm 0.022$, One-way ANOVA followed by Bonferroni's test, $n=2$, Fig. $4 \mathrm{a}$ and $\mathrm{b}$ ). 


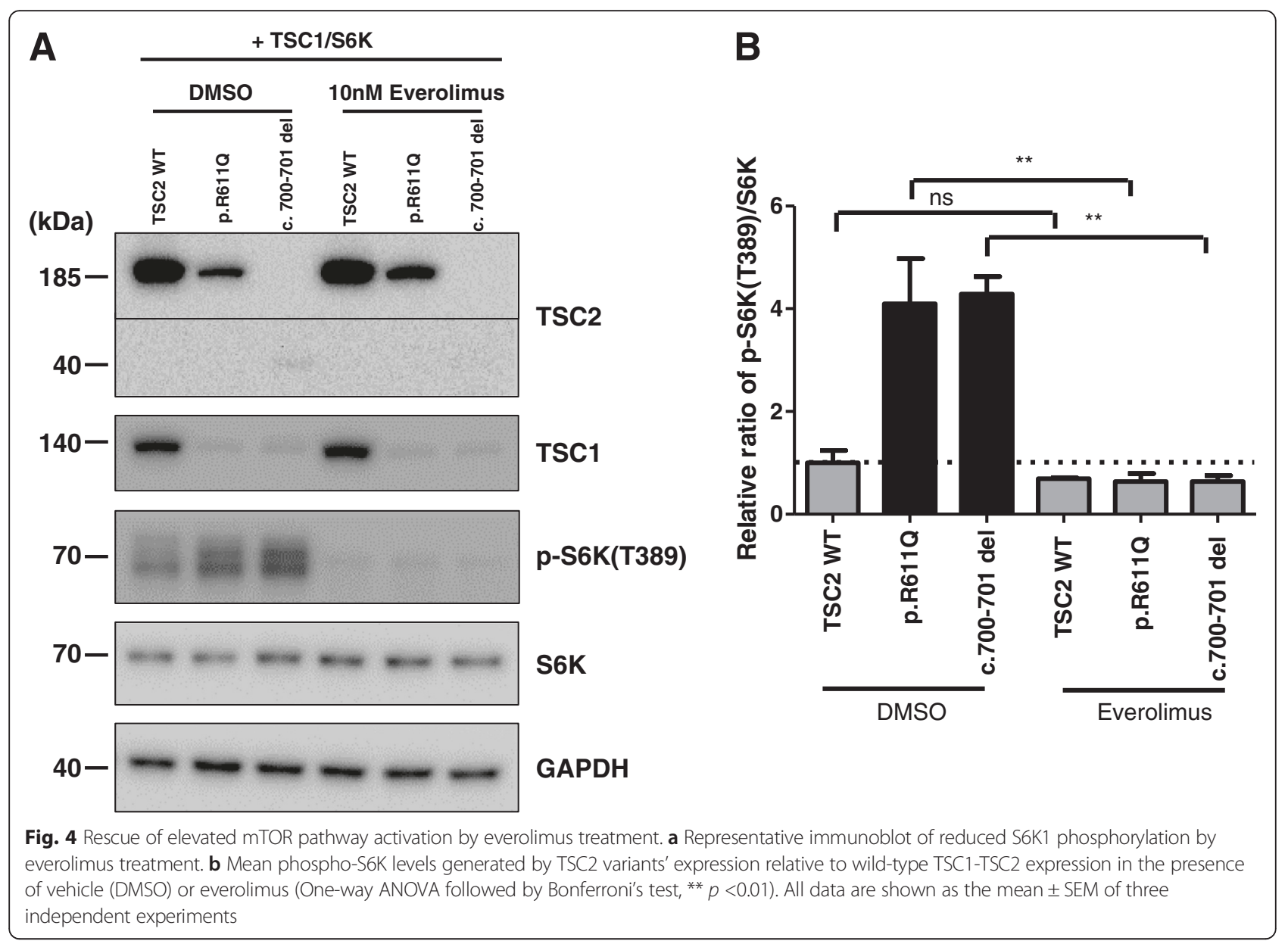

\section{Everolimus dosing and tolerability}

Confirming that the mutation caused hyperactivation of mTOR signaling encouraged us to treat the patient with the mTOR inhibitor everolimus. Everolimus was thus administered when the patient was 45 months of age. The body surface area was $0.86 \mathrm{~m}^{2}$ and the median maintenance dose of everolimus was $5.81 \mathrm{mg} /$ $\mathrm{m}^{2} /$ day. Serum levels during the maintenance dose were between 5 and $15 \mathrm{ng} / \mathrm{mL}$. During the study, the patient exhibited aggression and irritability for several weeks. No severe or life-threatening side effects related to treatment occurred. The patient recovered without dose adjustment and was able to continue everolimus treatment.

\section{Everolimus treatment reduced size of SEGA}

There was a size reduction of SEGA on post-treatment brain MRI after 4 months compared to baseline. Pretreatment coronal T2-weighted imaging shows a $1.5 \mathrm{~cm}$ sized hypointense SEGA located at the foramen of Monro (Fig. 1b). Post-treatment coronal T2-weighted imaging reveals a reduced size of SEGA with a maximum diameter of $1.1 \mathrm{~cm}$ (Fig. 1d).
Multiple cortical tubers and a subependymal nodule within on pre-treatment MRI (Fig. 1c) demonstrated reduced cortical tubers and the same size of subependymal nodule in post-treatment MRI (Fig. 1e).

\section{Everolimus treatment reduced seizure frequency and duration}

After treatment with everolimus, the seizure frequency and duration in the patient was significantly reduced. Vigabatrin and levetiracetam were withdrawn by the parents 1 month after treatment and the patient was seizure free for more than 3 months. Although seizures occasionally recurred during treatment with everolimus for which vigabatrin was administered, the median seizure frequency was decreased by $90 \%$ (pre- vs. post-treatment, 21 vs. 2 seizures per week) and the median seizure duration was also decreased by $98 \%$ (600 vs. 10 s) compared to that prior to everolimus treatment (Fig. 5a). In addition, electroencephalography (EEG) showed run of spikes in the right fronto-polar region prior to everolimus treatment, whereas no epileptiform discharge was observed following treatment (Fig. 5a). 


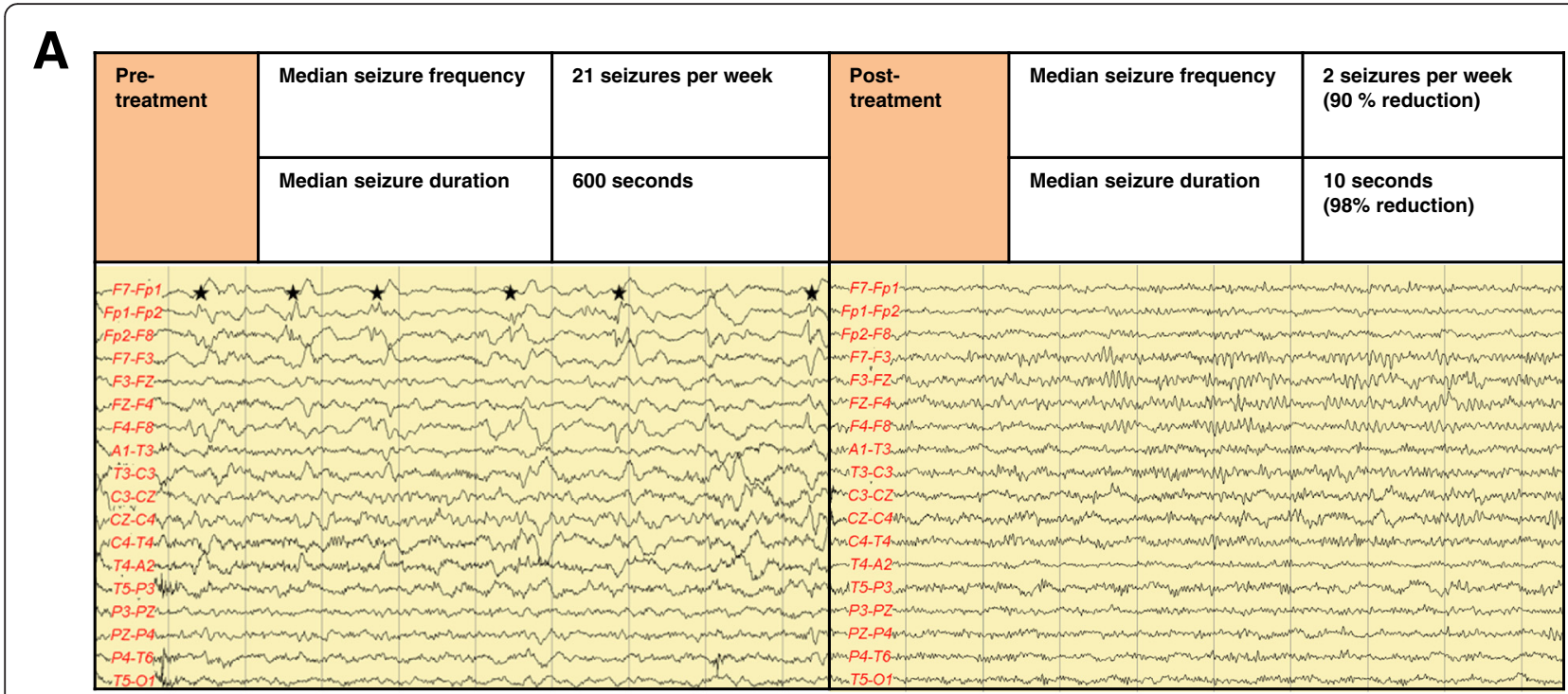

B

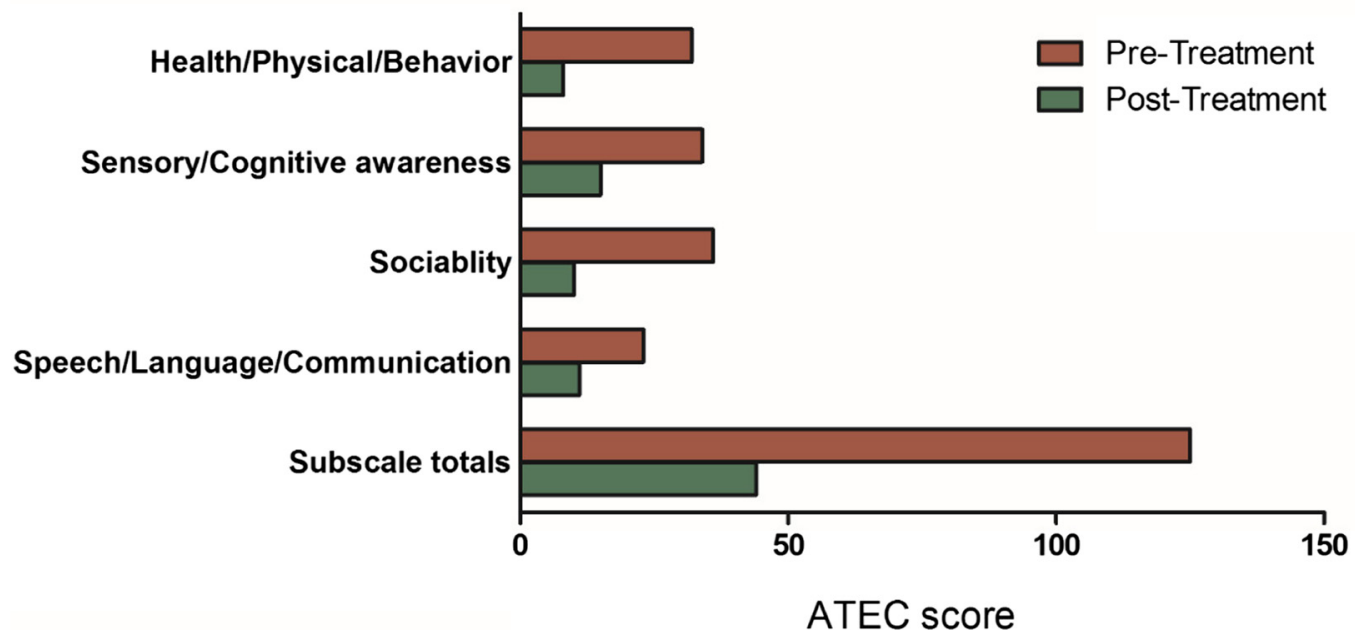

Fig. 5 Effect of everolimus treatment on seizure and general behavioral features. a The median seizure frequency and duration decreased compared to that prior to everolimus treatment. EEG showed run of spikes in the right fronto-polar region prior to everolimus treatment (left side), whereas no epileptiform discharge was observed following treatment (right side). $\mathbf{b}$ In the evaluation of the improvement in general and autistic behavioral features, ATEC showed a decrease in the scores in all four categories (Speech/Language/Communication, Sociability, Sensory/Cognitive awareness, and Health/Physical/Behavior) after everolimus treatment compared to pre-treatment

\section{Everolimus treatment improved general behavioral \\ deficits and autistic phenotypes}

Everolimus treatment had a great impact on reducing behavioral deficits and improved cognition, attention, social interaction, and language development in the patient. Just 1 day after the initiation of treatment, he started to understand and follow some verbal commands. After several days, episodes of urinary incontinence no longer occurred and the patient urinated in the toilet for the first time. Repetitive motions such as swaying or twirling and obsession with running water disappeared. At 1 month after treatment, the patient began to use two words sentences.
The parents were also asked to provide the Autism Treatment Evaluation Checklist (ATEC) scores prior to and after 1 year of the intervention. The ATEC has been successfully used to measure treatment effects and progress over time in several studies on ASD [32-34]. Following the initiation of treatment, the ATEC score was markedly decreased indicating a decrease in severity of ASD symptoms (Fig. 5b). Subscale totals more than 104 are considered to indicate very severe autism as they are in the 90th percentile; patients with scores less than 50 are considered to have good chances of being semiindependent and are within the 30th percentile level. A lowered score by over 50 points is considered to 
represent marked improvement following autism treatment. Here, the subscale totals of the patient decreased from 127 prior to treatment to 44 following treatment initiation; substantial decreases were observed following treatment initiation in all four of the ATEC categories. The Speech/Language/Communication ATEC scores decreased from 23 prior to treatment to 11 following treatment initiation; Sociability ATEC scores decreased from 36 to 10; Sensory/Cognitive Awareness ATEC scores decreased from 34 to 15; and the Health/Physical/Behavior ATEC scores decreased from 32 to 8 . The efficacy of everolimus on general and autistic behavioral deficit were not diminished over up to 2 days without medication, but it remains to be systematically determined how long the effect of everolimus would last following treatment cessation.

\section{Discussion}

In the present study, we describe a case of a Korean family in which three of the members have been diagnosed with TSC. Among the affected members, only a boy (III-2 in Fig. 2a) showed diverse behavioral and cognitive deficits including autistic phenotypes. Whole exome analysis revealed a novel deletion mutant in the TSC2 gene (TSC2 c.700-701 del).

We found that the expression of the newly identified mutant TSC2 protein enhanced the activation of the mTOR signaling pathway in HEK293T cells, suggesting that the novel mutation represents a loss-of-function mutation as are other TSC mutations associated with TSC [28]. In our biochemical analyses, we found that the protein expression level of the deletion mutant was significantly decreased (Fig. 3a, b). The reduced TSC2 c.700-701 del mutant expression level might be caused by nonsense-mediated mRNA decay as a quality-control mechanism [35-37]. In addition, it has been reported that the TSC1-TSC2 interaction is important for stability of both TSC1 and TSC2 [38, 39]. In this patient, it is hypothesized that the TSC1 protein would be destabilized since the mutation in TSC2 is located within the essential region for interacting with TSC1 (amino acids 1900) $[31,40]$ (Fig. 3a, c). Furthermore, the loss of the Cterminal GTPase activating protein (GAP) domain of TSC2 would also be predicted to disrupt the action of the TSC1-TSC2 complex on the GTPase Ras homolog expressed in brain (RHEB), thus activating RHEB-GTPdependent stimulation of the mammalian target of rapamycin complex 1 (mTORC1) [41]. Therefore, one of the downstream mTORC1 targets, S6K T389 phosphorylation, would be elevated (Fig. 3a, d). Additionally, we examined the effect of everolimus treatment on elevated S6K T389 phosphorylation induced by TSC2 c.700-701 del mutation and found that everolimus could reduce the activation of the mTOR signaling pathway (Fig. 4a, b).

The efficacy of mTOR inhibitors for treating TSCassociated phenotypes has been demonstrated in multiple animal models. For example, Ehninger and colleagues showed that rapamycin treatment reversed the deficits in learning and in hippocampal synaptic plasticity in $T s c 2^{+/-}$mice [18]. Rapamycin or everolimus (RAD001, 40-O-(2-hydroxyethyl)-rapamycin) treatment rescued lethality, brain enlargement, and hyperactivity in Tsc1 conditional knockout mice $[18,19]$. Notably, deleting Tsc1 in mouse cerebellar Purkinje cells resulted in autistic-like behaviors, which can also be reversed by rapamycin treatment [20]. In addition, deficits in social interaction in $T s c 1^{+/-}$and $T s c 2^{+/-}$mice were also reversed by rapamycin treatment [26]. These animal studies support the hypothesis that mTOR activation is responsible for the ASD-associated phenotypes in TSC $[16,26,42]$ and thereby mTOR inhibition might be an effective treatment strategy for ASD symptoms in TSC patients $[10,25]$.

In our study, everolimus treatment resulted in a rapid and marked reduction of behavioral deficits and improved cognition, attention, social interaction, and language development in the patient. As indicated by changes in the ATEC subscale scores, the classification of severe autism with scores in the 90th percentile was modified to that of semi-independent autism with scores in the 30th percentile level. The total score dropped by 83 points, which is considered to be a remarkable improvement for an autism treatment. In addition to the improvement of autistic features, everolimus showed marked effectiveness in mediating the intractable seizures of TSC, such that the seizure frequency and the median seizure duration decreased by over $90 \%$ in the patient. However, we cannot insist that everolimus would have therapeutic effect specifically on ASD or epilepsy in patients with TSC induced by TSC2 c.700-701 del mutation because grandmother and father carrying TSC2 c.700-701 del mutation has normal intelligence without epilepsy or neuropsychiatric symptoms and we could not suggest an evidence of genotype (TSC2 c.700701 del mutation)-phenotype correlation in TSC patient. Therefore, we consider that individuals affected by TSC2 c.700-701 del mutation could exhibit a high variability in clinical findings and further studies are needed to identify a key mechanism underlying the therapeutic effect of everolimus on TSC symptoms with ASD.

Nevertheless, early recognition of ASD in patients with TSC and proper management with everolimus might give a life-enhancing effect on the long-term outcome of the disorder. Consistent with our findings, Ishii and colleagues recently reported that everolimus treatment improved the irritability, stereotypic behavior, inappropriate speech, and 
social behavior in a 27 year-old female patient with TSC [43]. In addition, Wheless showed that everolimus reduced the seizure frequency in a 13-year-old girl with TSC-associated epilepsy after 1.5 years of treatment [44]. Kruger and colleagues also demonstrated the antiepileptic effect of everolimus in the majority of TSC patients [45]. Focal cortical malformations such as cortical tuber are highly associated with epileptogenesis and epilepsy related to cortical tuber is often refractory to antiepileptics [46]. mTOR inhibitor treatment also reduces the size of cortical tubers in TSC patients [47].

Together with our results, these studies strongly support that the inhibition of mTOR signaling represents a valid treatment strategy for the neurological and psychiatric manifestations associated with TSC. As additional clinical trials to test the efficacy of everolimus for treating the psychiatric symptoms associated with TSC have been completed or launched [10, 17], it will likely soon be determined whether the use of everolimus or other mTOR inhibitors will be approved to treat the features of ASD associated with TSC in the clinic.

In summary, we identified a novel small deletion mutation in TSC2 associated with severe TSC in a Korean family that enhances the activation of mTOR signaling in vitro. Moreover, everolimus treatment showed not only reduction in SEGA size, but improved behavioral deficits including autistic phenotypes and seizures in the patient.

\section{Methods}

\section{Participants}

The study participants consisted of a three-generation family whose members were diagnosed with TSC. TSC showed autosomal dominant inheritance throughout the family history; the grandmother was the first affected family member. Six individuals including the grandmother, father, mother, second and third sons, and daughter participated in this study.

\section{Diagnostic evaluation \\ Diagnosis of TSC}

Brain MRI, EEG, complete ophthalmologic evaluation including dilated fundoscopy, detailed dental examination, careful skin examination with a Wood's lamp, ultrasonography and ECG of the heart, HRCT of the chest, blood pressure, abdominal MRI, and GFR tests were performed. In addition to genetic diagnosis (described below), clinical diagnosis was made according to the "Tuberous Sclerosis Complex Diagnostic Criteria Update: Recommendations of the 2012 International Tuberous Sclerosis Complex Consensus Conference" [48].

\section{Measures and procedure}

A baseline psychomotor developmental evaluation including the DDST-II, WPPSI-III, CARS, SMS, and speech and language evaluations was performed prior to everolimus treatment. The ADOS and ADI-R were conducted by an experienced examiner. For ADOS, module 1 for 31 months and older children with pre-verbal/single words was applied. The ATEC was also implemented for the severity evaluation of autistic features prior to and following completion of the intervention.

\section{Intervention}

Everolimus treatment was initiated, at $5 \mathrm{mg} / \mathrm{m}^{2} /$ day, administered once daily in the morning and rounded to the nearest $2.5 \mathrm{mg} / \mathrm{dose}$. A serum everolimus level was obtained at every 2 weeks, and the treatment dose was adjusted to obtain a target range between 5 and $15 \mathrm{ng} /$ $\mathrm{ml}$. No further adjustments were made after the stable target range was obtained, unless a severe side effect or considerable weight gain was noticed. Safety and efficacy of treatment was observed for 1 year.

\section{Whole exome sequencing and variant calling}

Whole blood was obtained from the family members (the grandmother, father, mother, elder brother, and little sister of the patient) after informed consent for the protocol (KNUH 2013-07-011-004) guided by the KNUH IRB was obtained. Genomic DNA was extracted from the blood from each subject and about $3 \mu \mathrm{g}$ genomic DNA from each family member was subjected to library preparation and exome capture following the Agilent SureSelect Human All Exon v4 Illumina PairedEnd Sequencing Library Prep Protocol (Agilent Technologies, Santa Clara, CA, USA). The prepared sequencing library was used to perform sequencing on an Illumina HiSeq-2000 system (San Diego, CA, USA) as a 100 bp paired-end run. In each sample, about 51-65 million reads were generated. The raw sequencing reads were checked and trimmed using the sickle program (version 1.33) to ensure the quality of the raw reads. Processed reads were mapped to the reference human genome sequence GRCh37 using the Burrow-Wheeler Aligner (BWA, version 0.7.10) [49]. To reduce the potential bias problems caused by the sequencing processes, the mapped duplicated reads were marked using Picard (version 1.118). Insertion and realignment (INDEL) realignment and base quality recalibration were performed using GATK (version 3.2.2) [50]. The average of the mean target coverage was 65 and $85 \%$ of the target exome was covered to $20 \times$. Using the alignments, both small nucleotide variants (SNVs) and small INDELs were called by the GATK HaplotypeCaller and called variants were filtered using the GATK variant quality score recalibration process. Finally, for each family member, 84,356-86,901 variants were obtained. The variants were further filtered to exclude those included in dbSNP142, 1000 Genomes Project (Oct 2014), NHLBI- 
ESP project with 6500 exomes, or ExAC 65,000 exomes at the level of $5 \%$ minor allele frequency (MAF) by ANNOVAR [51]. In addition, variants were filtered out if they existed in in-house genome and exome databases.

\section{Sanger sequencing}

To validate the small deletion variant in the TSC2 gene, Sanger sequencing was performed. The target site of the variant and the flanking sequences of the DNA template from each family member were amplified with specific primers (forward primer 5'-ACAGTGACAGGGACGTCAGGTG-3' and reverse primer 5'-ACAACCATTCATGGGAGACAGGA-3') and the amplified products were directly sequenced on an ABI PRISM 3730 automated sequencer (Applied Biosystems, Foster City, CA, USA). The results were compared with the reference human genome sequence, GRCh37, to confirm the deletion variant.

\section{DNA constructs}

Full-length TSC1 with a Myc-tag at the C-terminus, wildtype TSC2, and the pathogenic TSC2 mutation p.R611Q were kindly provided by Dr. Mark Nellist (Eramus Medical Centre, The Netherlands). pcDNA-Myc S6K1 was a gift from Jie Chen (Addgene plasmid \#26610, Cambridge, MA, USA) [52]. The novel TSC2 c.700-701 deletion construct was derived from the wild-type TSC 2 construct by site-directed mutagenesis using a QuikChange II XL SiteDirected Mutagenesis Kit (Agilent Technologies, Santa Clara, CA). N-terminal HA-tagged wild-type TSC2, TSC2 p.R611Q, and TSC2 c.700-701 deletion mutants were subcloned into the pcDNA3.1(+) vector using BamHI and $X$ hoI sites. The complete open reading frame of the each construct was verified by sequencing. Construct DNA were prepared using the PureYield ${ }^{\mathrm{TM}}$ Plasmid Midiprep System (Promega, Madison, WI, USA).

\section{Antibodies}

Phospho-p70 S6 kinase Thr389 rabbit monoclonal (108D2 for Fig. 3 or 9205S for Fig. 4), Myc-tag mouse monoclonal (9B11), and HRP-conjugated anti-rabbit IgG (\#7074) antibodies were purchased from Cell Signaling Technology (Danvers, MA, USA). HRP-conjugated goat anti-rat IgG (\#AP183P), HRP-conjugated anti-mouse IgG (H+L) (\#SA001-500), and anti-HA-Fluorescein, high affinity (\#11 988506 001) antibodies were obtained from Millipore (Darmstadt, Germany), GenDEPOT (Barker, TX, USA), and Roche (Basel, Switzerland), respectively.

\section{Immunoblotting}

A transfection-based immunoblot assay for the functional assessment of the TSC2 variants was performed as described previously [30, 31]. HEK293T cells were plated on a 12 -well plate and grown overnight in Dulbecco's modified eagle's medium (DMEM) (Hyclone,
Logan, UT, USA) with $10 \%$ fetal bovine serum (FBS). Confluent cells (70-90\%) were transfected with $0.4 \mu \mathrm{g}$ TSC2, $0.8 \mu \mathrm{g}$ TSC1, and 0.2-0.3 $\mu \mathrm{g}$ S6K1 constructs using $4 \mu \mathrm{L}$ Lipofectamine 2000 (Thermo Fisher Scientific, Waltham, MA, USA) in Opti-MEM. The transfected medium was replaced with DMEM with $10 \%$ FBS at $4 \mathrm{~h}$ after transfection. And then, everolimus (10 $\mathrm{nM}$, LC Laboratories, Woburn, MA, USA) was added and incubated for another $20 \mathrm{~h}$. The transfected cells were harvested, washed with cold phosphate-buffered saline, and lysed with $200 \mu \mathrm{L}$ RIPA buffer $(150 \mathrm{mM} \mathrm{NaCl}, 1.0 \%$ Triton X-100, $0.5 \%$ sodium deoxycholate, $0.1 \%$ SDS, and $50 \mathrm{mM}$ Tris- $\mathrm{Cl}, \mathrm{pH}$ 8.0) containing a protease inhibitor cocktail and a phosphatase inhibitor cocktail (Roche). After full-speed centrifugation for $15 \mathrm{~min}$ at $4{ }^{\circ} \mathrm{C}$, the supernatant fractions were quantified using a Thermo Scientific $^{\mathrm{TM}}$ Pierce $^{\mathrm{TM}}$ BCA Protein Assay kit. The protein samples $\left(4.5 \mu \mathrm{g}\right.$ each) were electrophoresed on Bolt ${ }^{\oplus} 4-$ $12 \%$ Bis-Tris Plus Gels (Thermo Fisher Scientific) and transferred to PVDF membranes (Millipore) according to the manufacturer's recommendations.

The blots were blocked for $10 \mathrm{~min}$ at room temperature with either $5 \%$ Blotto, non-fat dry milk (sc-2325, Santa Cruz Biotechnology, Dallas, TX, USA) or $5 \%$ bovine serum albumin in TBST (Tris-buffered saline plus $0.1 \%$ Tween-20). Blots were incubated overnight at $4{ }^{\circ} \mathrm{C}$ with the following primary antibodies: 1:1000 dilutions of rabbit monoclonal anti-p-S6K (T389) or rat anti-HA antibody, or 1:10,000 dilutions of mouse monoclonal anti-myc tag antibody. After washing three times for $10 \mathrm{~min}$ in TBST, the blots were incubated for $2 \mathrm{~h}$ at room temperature with 1:5000 dilutions of secondary antibodies. After washing three times for $10 \mathrm{~min}$ in TBST, the blots were scanned using a ChemiDoc ${ }^{\mathrm{TM}}$ XRS System (Bio-Rad, Hercules, CA, USA) after applying detection reagents. To estimate the ratio of p-S6K (T389) to total S6K, the scans were analyzed using Image $\mathrm{Lab}^{\mathrm{TM}}$ Software (Bio-Rad).

\section{Statistics}

One-way analysis of variance (ANOVA) with appropriate post-hoc tests (Dunnett's or Bonferroni's test) was used for western blotting results. Each " $n$ " indicates an independent set of experiment. Differences are considered significant at the level of $p<0.05$.

\section{Additional file}

Additional file 1: Table S1. A list of identified variants that were not detected in unaffected members but detected in affected members (PDF $35 \mathrm{~kb}$ )

\section{Abbreviations}

ASD: autism spectrum disorder; TSC: tuberous sclerosis; mTOR: mammalian target of rapamycin; SEGA: subependymal giant cell astrocytomas;

MRI: magnetic resonance image; DDST-II: Denver Developmental Screening 
Test; WPPSI-III: Wechsler Preschool and Primary Scale of Intelligence; SMS: Social Maturity Scale; CARS: Childhood Autism Rating Scale; ADOS2: Autism Diagnostic Observation Schedule-2; ADI-R: Autism Diagnostic Interview-Revised; ECG: electrocardiography; HRCT: high resolution computed tomography; GFR: glomerular filtration rate; EEG: electroencephalography; ATEC: Autism Treatment Evaluation Checklist; GAP: GTPase activating protein; RHEB: Ras homolog expressed in brain; mTORC1: mammalian target of rapamycin complex 1; INDELs: insertions and deletions; SNVs: small nucleotide variants; MAF: minor allele frequency; DMEM: Dulbecco's modified eagle's medium.

\section{Acknowledgements}

We thank the patient and family who participated in this study and Dr. Mark Nellist for sharing the plasmids for TSC1, TSC2, and TSC2-R611Q

\section{Funding}

This work was supported by the National Honor Scientist Program to B.K.K (NRF-2012R1A3A1050385).

\section{Authors' contributions}

$\mathrm{SKH}$ and $\mathrm{KL}$ participated in the study design and discussions, performed the research, recruited and enrolled patients, oversaw data collection, and collected and interpreted the data. JEY performed the biochemistry experiments. JHL analyzed the exome sequencing data. SKH, KL, JHL, CSL, JAL, YSL, and BKK performed the literature review and wrote the manuscript. All authors read and approved the final manuscript.

\section{Competing interests}

The authors declare that they have no competing interests.

\section{Consent for publication}

Written informed consent to publish was obtained from the patient and the parents.

\section{Ethics approval and consent to participate}

The Kyungpook National University Hospital Institutional Review Board (KNUH IRB) approved the intervention protocol (KNUH 2013-07-011-004), and the safety of clinical intervention was monitored regularly by independent reviewers in KNUH IRB. Informed consent was obtained from the patients or their parent.

\section{Author details}

'Department of Pediatrics, Kyungpook National University Hospital, Daegu 41944, South Korea. ${ }^{2}$ Department of Life and Nanopharmaceutical Sciences, Department of Maxillofacial Biomedical Engineering, School of Dentistry, Kyung Hee University, Seoul 02447, South Korea. ${ }^{3}$ Department of Biological Sciences, College of Natural Sciences, Seoul National University, Seoul 08826, South Korea. ${ }^{4}$ Department of Biotechnology and Biological Sciences, Hannam University, Daejeon 34430, South Korea. ${ }^{5}$ Department of Life Science, Chung-Ang University, Seoul 06974, South Korea. ${ }^{6}$ Behavioral Neural Circuitry and Physiology Laboratory, Department of Anatomy, Brain Science \& Engineering Institute, Kyungpook National University Graduate School of Medicine, Daegu 41944, South Korea.

Received: 15 January 2016 Accepted: 9 April 2016

Published online: 23 May 2016

\section{References}

1. Crino PB, Nathanson $\mathrm{KL}$, Henske EP. The tuberous sclerosis complex. N Engl J Med. 2006;355:1345-56.

2. Peters JM, Taquet M, Vega C, Jeste SS, Fernandez IS, Tan J, Nelson CA, 3rd, Sahin M, Warfield SK. Brain functional networks in syndromic and nonsyndromic autism: a graph theoretical study of EEG connectivity. BMC Med. 2013;11:54.

3. Osborne JP, Fryer A, Webb D. Epidemiology of tuberous sclerosis. Ann N Y Acad Sci. 1991:615:125-7.

4. Franz DN, Bissler JJ, McCormack FX. Tuberous sclerosis complex: neurological, renal and pulmonary manifestations. Neuropediatrics. 2010;41: 199-208.

5. Krueger DA, Northrup $\mathrm{H}$, International Tuberous Sclerosis Complex Consensus G. Tuberous sclerosis complex surveillance and management: recommendations of the 2012 International Tuberous Sclerosis Complex Consensus Conference. Pediatr Neurol. 2013:49:255-65.

6. Gillberg IC, Gillberg C, Ahlsen G. Autistic behaviour and attention deficits in tuberous sclerosis: a population-based study. Dev Med Child Neurol. 1994; 36:50-6.

7. Joinson C, O'Callaghan FJ, Osborne JP, Martyn C, Harris T, Bolton PF. Learning disability and epilepsy in an epidemiological sample of individuals with tuberous sclerosis complex. Psychol Med. 2003;33:335-44.

8. Jeste SS, Sahin M, Bolton P, Ploubidis GB, Humphrey A. Characterization of autism in young children with tuberous sclerosis complex. J Child Neurol. 2008;23:520-5.

9. Curatolo P, Porfirio MC, Manzi B, Seri S. Autism in tuberous sclerosis. Eur J Paediatr Neurol. 2004:8:327-32

10. Ehninger D. From genes to cognition in tuberous sclerosis: implications for mTOR inhibitor-based treatment approaches. Neuropharmacology. 2013;68: 97-105.

11. van Slegtenhorst $M$, de Hoogt $R$, Hermans $C$, Nellist $M$, Janssen $B$, Verhoef S, Lindhout D, van den Ouweland A, Halley D, Young J, Burley M, Jeremiah S, Woodward K, Nahmias J, Fox M, Ekong R, Osborne J, Wolfe J, Povey S, Snell RG, Cheadle JP, Jones AC, Tachataki M, Ravine D, Sampson JR, Reeve MP, Richardson P. Wilmer F, Munro C, Hawkins $T L$, et al. Identification of the tuberous sclerosis gene TSC1 on chromosome 9q34. Science. 1997;277:805-8.

12. Au KS, Williams AT, Roach ES, Batchelor L, Sparagana SP, Delgado MR, Wheless JW, Baumgartner JE, Roa BB, Wilson CM, Smith-Knuppel TK, Cheung MY, Whittemore VH, King TM, Northrup H. Genotype/phenotype correlation in 325 individuals referred for a diagnosis of tuberous sclerosis complex in the United States. Genet Med. 2007:9:88-100.

13. Jones AC, Daniells CE, Snell RG, Tachataki M, Idziaszczyk SA, Krawczak M, Sampson JR, Cheadle JP. Molecular genetic and phenotypic analysis reveals differences between TSC1 and TSC2 associated familial and sporadic tuberous sclerosis. Hum Mol Genet. 1997:6:2155-61.

14. Dabora SL, Jozwiak S, Franz DN, Roberts PS, Nieto A, Chung J, Choy YS, Reeve MP, Thiele E, Egelhoff JC, Kasprzyk-Obara J, Domanska-Pakiela D, Kwiatkowski DJ. Mutational analysis in a cohort of 224 tuberous sclerosis patients indicates increased severity of TSC2, compared with TSC1, disease in multiple organs. Am J Hum Genet. 2001;68:64-80.

15. Kwiatkowski DJ, Manning BD. Tuberous sclerosis: a GAP at the crossroads of multiple signaling pathways. Hum Mol Genet. 2005;14(Spec No. 2):R251-258.

16. de Vries PJ. Targeted treatments for cognitive and neurodevelopmental disorders in tuberous sclerosis complex. Neurotherapeutics. 2010;7:275-82.

17. Davis PE, Peters JM, Krueger DA, Sahin M. Tuberous sclerosis: a new frontier in targeted treatment of autism. Neurotherapeutics. 2015;12:572-83.

18. Ehninger D, Han S, Shilyansky C, Zhou Y, Li W, Kwiatkowski DJ, Ramesh V, Silva AJ. Reversal of learning deficits in a Tsc2+/- mouse model of tuberous sclerosis. Nat Med. 2008;14:843-8.

19. Meikle L, Pollizzi K, Egnor A, Kramvis I, Lane H, Sahin M, Kwiatkowski DJ. Response of a neuronal model of tuberous sclerosis to mammalian target of rapamycin (mTOR) inhibitors: effects on mTORC1 and Akt signaling lead to improved survival and function. J Neurosci. 2008;28:5422-32.

20. Tsai PT, Hull C, Chu Y, Greene-Colozzi E, Sadowski AR, Leech JM, Steinberg J, Crawley JN, Regehr WG, Sahin M. Autistic-like behaviour and cerebellar dysfunction in Purkinje cell Tsc1 mutant mice. Nature. 2012:488:647-51.

21. Krueger DA, Care MM, Holland K, Agricola K, Tudor C, Mangeshkar P, Wilson KA, Byars A, Sahmoud T, Franz DN. Everolimus for subependymal giant-cell astrocytomas in tuberous sclerosis. N Engl J Med. 2010;363:1801-11.

22. Hofbauer GF, Marcollo-Pini A, Corsenca A, Kistler AD, French LE, Wuthrich $R P$, Serra AL. The mTOR inhibitor rapamycin significantly improves facial angiofibroma lesions in a patient with tuberous sclerosis. Br J Dermatol. 2008;159:473-5.

23. Davies DM, de Vries PJ, Johnson SR, McCartney DL, Cox JA, Serra AL, Watson PC, Howe CJ, Doyle T, Pointon K, Cross JJ, Tattersfield AE Kingswood JC, Sampson JR. Sirolimus therapy for angiomyolipoma in tuberous sclerosis and sporadic lymphangioleiomyomatosis: a phase 2 trial. Clin Cancer Res. 2011;17:4071-81

24. Bissler JJ, McCormack FX, Young LR, Elwing JM, Chuck G, Leonard JM, Schmithorst VJ, Laor T, Brody AS, Bean J, Salisbury S, Franz DN. Sirolimus for angiomyolipoma in tuberous sclerosis complex or lymphangioleiomyomatosis. N Engl J Med. 2008;358:140-51.

25. Ehninger D, Silva AJ. Rapamycin for treating Tuberous sclerosis and Autism spectrum disorders. Trends Mol Med. 2011;17:78-87. 
26. Sato A, Kasai S, Kobayashi T, Takamatsu Y, Hino O, Ikeda K, Mizuguchi M. Rapamycin reverses impaired social interaction in mouse models of tuberous sclerosis complex. Nat Commun. 2012;3:1292.

27. Javelaud D, Alexaki VI, Dennler S, Mohammad KS, Guise TA, Mauviel A. TGFbeta/SMAD/GLI2 signaling axis in cancer progression and metastasis. Cancer Res. 2011;71:5606-10.

28. Orlova KA, Crino PB. The tuberous sclerosis complex. Ann N Y Acad Sci. 2010;1184:87-105.

29. Wang GX, Wang DW, Yi CY, Qu JS, Wang YL. Mutational analyses of the TSC1 and TSC2 genes in cases of tuberous sclerosis complex in Chinese Han children. Genet Mol Res. 2013;12:1168-75.

30. Hoogeveen-Westerveld M, Ekong R, Povey S, Mayer K, Lannoy N, Elmslie F, Bebin M, Dies K, Thompson C, Sparagana SP, Davies P, van Eeghen AM, Thiele EA, van den Ouweland A, Halley D, Nellist M. Functional assessment of TSC2 variants identified in individuals with tuberous sclerosis complex. Hum Mutat. 2013;34:167-75.

31. Hoogeveen-Westerveld $M$, Wentink $M$, van den Heuvel $D$, Mozaffari $M$, Ekong R, Povey S, den Dunnen JT, Metcalfe K, Vallee S, Krueger S, Bergoffen J, Shashi V, Elmslie F, Kwiatkowski D, Sampson J, Vidales C, Dzarir J, GarciaPlanells J, Dies K, Maat-Kievit A, van den Ouweland A, Halley D, Nellist M. Functional assessment of variants in the TSC1 and TSC2 genes identified in individuals with Tuberous Sclerosis Complex. Hum Mutat. 2011;32:424-35.

32. Magiati I, Moss J, Yates R, Charman T, Howlin P. Is the Autism Treatment Evaluation Checklist a useful tool for monitoring progress in children with autism spectrum disorders? J Intellect Disabil Res. 2011;55:302-12.

33. Lonsdale D, Shamberger RJ, Audhya T. Treatment of autism spectrum children with thiamine tetrahydrofurfuryl disulfide: a pilot study. Neuro Endocrinol Lett. 2002;23:303-8.

34. Geier DA, Kern JK, Geier MR. A Comparison of the Autism Treatment Evaluation Checklist (ATEC) and the Childhood Autism Rating Scale (CARS) for the quantitative evaluation of autism. J Ment Health Res Intellect Disabil. 2013:6:255-67.

35. Chang YF, Imam JS, Wilkinson MF. The nonsense-mediated decay RNA surveillance pathway. Annu Rev Biochem. 2007;76:51-74.

36. Hentze MW, Kulozik AE. A perfect message: RNA surveillance and nonsensemediated decay. Cell. 1999;96:307-10.

37. Holbrook JA, Neu-Yilik G, Hentze MW, Kulozik AE. Nonsense-mediated decay approaches the clinic. Nat Genet. 2004;36:801-8.

38. Benvenuto G, Li S, Brown SJ, Braverman R, Vass WC, Cheadle JP, Halley DJ, Sampson JR, Wienecke R, DeClue JE. The tuberous sclerosis-1 (TSC1) gene product hamartin suppresses cell growth and augments the expression of the TSC2 product tuberin by inhibiting its ubiquitination. Oncogene. 2000; 19:6306-16.

39. Chong-Kopera H, Inoki K, Li Y, Zhu T, Garcia-Gonzalo FR, Rosa JL, Guan KL. TSC1 stabilizes TSC2 by inhibiting the interaction between TSC2 and the HERC1 ubiquitin ligase. J Biol Chem. 2006;281:8313-6.

40. Nellist M, Verhaaf B, Goedbloed MA, Reuser AJ, van den Ouweland AM, Halley DJ. TSC2 missense mutations inhibit tuberin phosphorylation and prevent formation of the tuberin-hamartin complex. Hum Mol Genet. 2001; 10:2889-98.

41. Huang J, Manning BD. The TSC1-TSC2 complex: a molecular switchboard controlling cell growth. Biochem J. 2008;412:179-90.

42. de Vries PJ, Howe CJ. The tuberous sclerosis complex proteins-a GRIPP on cognition and neurodevelopment. Trends Mol Med. 2007;13:319-26.

43. Ishii R, Wataya-Kaneda M, Canuet L, Nomomura N, Nakai Y, Takeda M. Everolimus improves behavioral deficits in a patient with autism associated with tuberous sclerosis: a case report. Neuropsychiatric Electrophysiology. 2015;1:6.

44. Wheless JW. Use of the mTOR inhibitor everolimus in a patient with multiple manifestations of tuberous sclerosis complex including epilepsy. Epilepsy Behav Case Rep. 2015;4:63-6.

45. Krueger DA, Wilfong AA, Holland-Bouley K, Anderson AE, Agricola K, Tudor C, Mays M, Lopez CM, Kim MO, Franz DN. Everolimus treatment of refractory epilepsy in tuberous sclerosis complex. Ann Neurol. 2013;74:679-87.

46. Ryther RC, Wong M. Mammalian target of rapamycin (mTOR) inhibition: potential for antiseizure, antiepileptogenic, and epileptostatic therapy. Curr Neurol Neurosci Rep. 2012;12:410-8.

47. Kim HS, Kim ST, Kang SH, Sung DJ, Kim CH, Shin SW, Kim YH, Cho WY, Park $\mathrm{KH}$. The use of everolimus to target carcinogenic pathways in a patient with renal cell carcinoma and tuberous sclerosis complex: a case report. J Med Case Rep. 2014;8:95.
48. Northrup H, Krueger DA, International Tuberous Sclerosis Complex Consensus G. Tuberous sclerosis complex diagnostic criteria update: recommendations of the 2012 linternational Tuberous Sclerosis Complex Consensus Conference. Pediatr Neurol. 2013:49:243-54.

49. Li H, Durbin R. Fast and accurate short read alignment with BurrowsWheeler transform. Bioinformatics. 2009;25:1754-60.

50. McKenna A, Hanna M, Banks E, Sivachenko A, Cibulskis K, Kernytsky A, Garimella K, Altshuler D, Gabriel S, Daly M, DePristo MA. The Genome Analysis Toolkit: a MapReduce framework for analyzing next-generation DNA sequencing data. Genome Res. 2010;20:1297-303.

51. Wang K, Li M, Hakonarson H. ANNOVAR: functional annotation of genetic variants from high-throughput sequencing data. Nucleic Acids Res. 2010;38:e164.

52. Fang Y, Vilella-Bach M, Bachmann R, Flanigan A, Chen J. Phosphatidic acidmediated mitogenic activation of mTOR signaling. Science. 2001;294:1942-5.

\section{Submit your next manuscript to BioMed Central and we will help you at every step:}

- We accept pre-submission inquiries

- Our selector tool helps you to find the most relevant journal

- We provide round the clock customer support

- Convenient online submission

- Thorough peer review

- Inclusion in PubMed and all major indexing services

- Maximum visibility for your research

Submit your manuscript at www.biomedcentral.com/submit
C Biomed Central 